\title{
Diálogos Digitais: Integração Entre Dados Documentais em Sistemas de Informação Baseados no Modelo para Conservação do Patrimônio Arquitetônico
}

\author{
Digital Dialogues: Integration of Documentary Data in Model-Based Information Systems for the \\ conservation of the architectural heritage
}

\author{
Eloisa Dezen-Kempter \\ Universidade Estadual de Campinas, Brasil \\ elo@ft.unicamp.br
}

\begin{abstract}
The architectural documentation plays a key role in the preservation of built heritage, as both support decision making in intervention projects, restoration and rehabilitation, such as the actions of preventive conservation. Building Information Modeling seems to be the adequate environment to assemble huge amounts of data. The aim of this research is to develop the integration of quantitative data (smart objects, performance data) and qualitative (photographs and historical documents) in model-based information, such as BIM, to be used in surveying, planning and control of interventions in historic buildings.
\end{abstract}

Keywords: BIM; Architectural Conservation databases; Interoperability.

\section{Introdução}

A documentação arquitetônica desempenha um papel essencial no processo de preservação do patrimônio histórico construído, pois apoia tanto as tomadas de decisão em projetos de intervenção, restauro e reabilitação, como as ações de conservação preventiva.

A introdução de novas tecnologias digitais no domínio do patrimônio cultural, como os sistemas de informação baseados no modelo, poderá representar a oportunidade de criar modelos tridimensionais como ferramentas de comunicação eficazes e intuitivas, com interfaces de compartilhamento e visualização tanto das características físico-estruturais da edificação como de seu inventário histórico.

Manferdini e Remondino (2012) colocam que os modelos geométricos tridimensionais, para documentação e preservação do patrimônio arquitetônico, apresentam-se como recursos mais vantajosos em relação às representações bidimensionais, por permitirem uma comunicação mais efetiva do real e atual aspecto físico da edificação, com suas deformações e patologias, através de uma interface mais intuitiva, rápida e atraente. Afirma ainda que, dependendo da tecnologia utilizada, poderá possibilitar também a obtenção de outros tipos de informações que vão além da geometria, como dados históricos, estado de conservação, materiais e técnicas construtivas adotadas.

Apesar das potencialidades do uso de sistemas de informação baseados no modelo na área de patrimônio arquitetônico, a experiência da aplicação da tecnologia BIM (Building Information Modeling) nesta área ainda é incipiente. Experiências do uso da tecnologia BIM aliada ao patrimônio histórico podem ser conferidas nos trabalhos de Stephen Fai et al (2011), Arayici (2008), Penttila et al (2007), Manferdini e Remondino (2012), Mokhtar Noriega et al (2011) entre outros.

A Tecnologia BIM não é apenas uma nova fórmula para a representação de novas propostas de construção, mas pode ser utilizado também como um banco de dados de informações de construção, auxiliando arquitetos e construtores para representar edifícios construídos e atuar em sua manutenção e monitoramento do seu ciclo de vida, controlando e medindo o desempenho das edificações com o apoio da inspeções periódicas e dispositivos de monitorização integrados (Mokhtar Noriega, 2011).

Fai et al. (2011) investigaram a construção de modelos de informação de construção, de edifícios de interesse cultural, que incorporam tanto informações quantitativas (objetos inteligentes, dados de desempenho) quanto as qualitativas (fotografias históricas, histórias orais, música). O modelo de informação de construção elaborado pelos autores para um grupo de edifícios do século XIX em Ontario, Canada, reúne séries de dados e tipos de dados heterogêneos em um único objeto digital que permite o acesso e verificação contínua dos dados individuais que compõem o todo. Isso inclui dados relativos ao tipo de edifício, construção e desempenho, bem como o material tipicamente associado com o patrimônio imaterial, como história oral e música.

Se atualmente análises laboratoriais cobrem e interpretam o testemunho material das edificações, o uso de novas tecnologias 
da informação, como a modelagem da informação da construção (BIM), colabora para o entendimento formal, estrutural e morfológico do objeto, servindo para organizar e disseminar a informação documental do bem patrimonial, unificando as informações métrico-arquitetônicas com a caracterização dos materiais.

No modelo de informação da construção são modelados todos os assuntos relativos ao edifício como os produtos, processos e documentos, servindo como um repositório único e organizado das diversas fontes de informações de uma edificação. Assim, o modelo torna-se o reflexo de todos os elementos que o geraram. As principais atividades relacionadas à modelagem tridimensional baseada no objeto, que foram descritas no trabalho "3D Working Method": (1) documentação; (2) simulação; (3) visualização; (4) controle de interferências; (5) intercâmbio e (6) extração de dados, estão esquematizadas na figura 1.

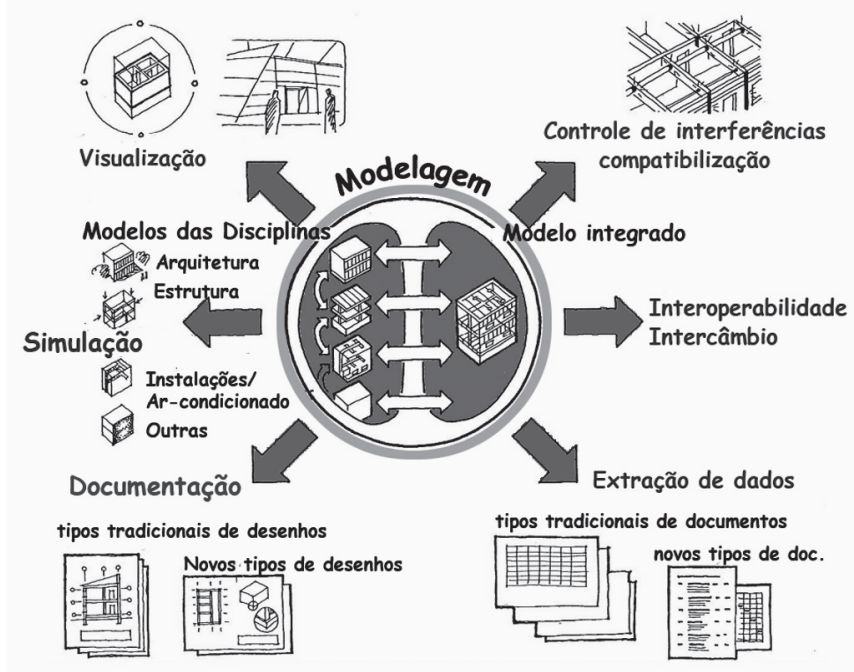

Figura 1: O método de trabalho em 3D. Fonte: BIS, 2008. Tradução da autora..

O presente artigo relata um projeto de pesquisa em andamento na Universidade Estadual de Campinas que tem como objetivo principal desenvolver um processo de integração de dados quantitativos (objetos inteligentes, dados de desempenho) e qualitativos (fotografias e documentos históricos) em sistemas de informação baseada no modelo, como o BIM para ser usado no levantamento, planejamento e controle das intervenções em edifícios históricos. A pesquisa tem como base exploratória da metodologia proposta o prédio da Cia Prada Indústria e Comércio, construído em 1937 em Limeira.

\section{O Patrimônio da Industrialização}

Somente recentemente as edificações industriais passaram a figurar como patrimônio cultural ao lado de outros tipos arquitetônicos.
O patrimônio industrial, quando se torna alvo de reabilitação, geralmente é percebido em um leque inconstante de prioridades, ações e agentes interventores. Essa variedade de abordagem está intimamente ligada às especificidades de escala, composição física e dinâmica evolutiva do tecido urbano em que está implantado, assim como se liga a fatores de restrições das entidades de salvaguarda patrimonial, de intenções políticas, de processos avaliativos, de pressão imobiliária, e muitas vezes à expectativa da própria comunidade.

Esforços imensos de acadêmicos, comunidades, organizações não governamentais, entre outros, têm sido feitos para preservar, inventariar e promover a reutilização respeitosa dos monumentos industriais.

Muitos destes antigos espaços fabris têm se tornado alvo de reabilitação, devido as suas especificidades formais e de escala. A intervenção em patrimônio histórico arquitetônico apresenta-se como um campo de domínio multidisciplinar, congregando áreas e profissionais de formações diversificadas. Como resultado, os projetos de restauro e reabilitação, a partir de pesquisas e abordagens em diversas áreas como arqueologia da arquitetura, história, tecnologia da construção, tecnologia dos materiais, ciências aplicadas, aliados à aplicação de tecnologias computacionais, prospecção e em acordo com os princípios internacionais de conservação e da teoria do restauro, tornam-se instrumentos fundamentais de intervenção no bem patrimonial.

Atualmente, o modelo tridimensional tem se tornado peça importante no processo de intervenção em patrimônio histórico, pois permite a compreensão do projeto arquitetônico, assim como o desenvolvimento de estudos e análises do caráter tectônico destas edificações. Ter acesso a uma fonte de informação que contemple as peculiaridades dos componentes arquitetônicos (fundação, estruturas, telhado, escadas, caixilharia, acabamentos e ornamento arquitetônico) que caracterizam as edificações industriais, assim como os seus aspectos históricos, torna-se fundamental para o planejamento criterioso do processo de reabilitação.

\section{O Edifício Prada}

O Edifício Prada foi construído em 1938 para abrigar as instalações da Fábrica de Chapéus Prada, fundada em 1907. O edifício, com tendência decó, enquanto solução arquitetônica e construtiva, tinha por objetivo obter a maior otimização do espaço e da funcionalidade.

A busca da máxima iluminação natural é evidenciada pela ampla distribuição de janelas ao longo das fachadas e da iluminação zenital (através de sheds e lanternins).

A flexibilidade de ocupação foi permitida pela sua modulação estrutural que graças ao uso do concreto armado possibilitou grandes vãos. Os pés-direitos altos contribuíam para a correta instalação dos equipamentos que transmitiam a força motriz. 


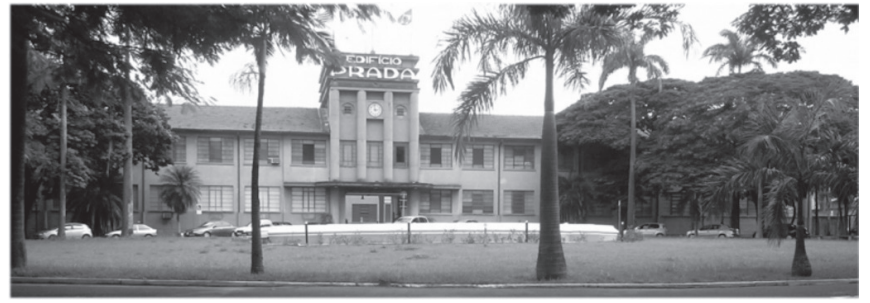

Figura 2: Fachada principal do Edifício Prada. Foto: Rafaela do Amarante Carneiro, 2013.

\section{Gerenciamento do Acervo Documental da Edificação}

O Objetivo dessa pesquisa em andamento é testar a interoperabilidade entre BIM e os dados documentais (fotos, textos, etc).

Está sendo realizado o levantamento e recuperação de toda documentação histórica da edificação, sua classificação e ranking e sua conversão em padrões para serem associados ao modelo empregando o IFC (industry foundation classes). Está sendo utilizado o Sistema de Classificação proposto na NBR 12006-2, cuja proposta é ordenar e hierarquizar a construção, dividindo-a por classes e/ou princípios de especialização, agrupando-os de forma coerente e de fácil entendimento.

Vários autores vêm trabalhando no desenvolvimento de métodos de integração de informações contidas em documentos do projeto de construção, de modo a poder explorar as características internas desses documentos e adaptando-os a diferentes projetos. Kosovac et al.(2000) propôs o uso de um vocabulário controlado por módulos e organizados de forma hierárquica em linguagem XML (extensible Markup Language). Wood (2000) apresenta uma abordagem para extração de estruturas hierárquicas de conceitos a partir de documentação textual do projeto. Zhu et al. (2001) propõe uma integração front-end baseado na Web com base na tecnologia XML.

Neste projeto de pesquisa estamos avaliando qual o melhor método aplicável para acervos de edificações históricas.

\section{Integração das Informações Padronizadas no Modelo 3D Documental da Edificação}

De posse dos dados identificados, classificados e hierarquizados, ocorrerá seu detalhamento através dos modelos tridimensionais. Os recursos utilizados na pesquisa incluem o aplicativo 123D Catch da Autodesk, para a criação de modelos digitais de malha triangular irregular a partir de uma série de fotos dos componentes da edificação. Na sequência, foram gerados modelos BIM no Revit da Autodesk, a partir da informação documental contida no projeto e das nuvens de pontos produzidas pelo $123 \mathrm{D}$
Catch, neste caso empregando-se o plug-in Scan to BIM (Imaginit Technologies).

Por fim será feita a associação de documentos não gráficos (textuais, iconográficos e em outras mídias) no modelo 3D. Será usada a metodologia "Text Information Integration Methodology Model" (TIIMM) desenvolvida por Caldas, Soibelman, Gasser (2005).

Essa metodologia é baseada primeiramente na classificação automatizada dos documentos da edificação. Essa classificação é realizada usando algoritmos de classificação de padrões. Os principais componentes do processo de classificação de documentos incluem: coleta de dados, conversão de dados, redução de dimensionalidade, preparação de dados, transformação de dados, aprendizado e classificação de documentos (Caldas, Soibelman, 2003).

A segunda etapa consiste na recuperação e ranking dos documentos classificados.

A Associação de documentos não gráficos aos modelos 3D do edifício é a última etapa da metodologia TIIMM. Depois que os documentos do projeto forem classificados e recuperados, uma referência aos documentos relevantes será adicionada ao modelo. Caldas e Soibelman (2003) alertam para o fato que os documento não gráficos não são adicionados ao modelo, mas apenas uma referência a eles. Isso facilita atualizações futuras, economiza espaço de armazenamento e memória do computador, e ajuda a integração com os sistemas de informação existentes.

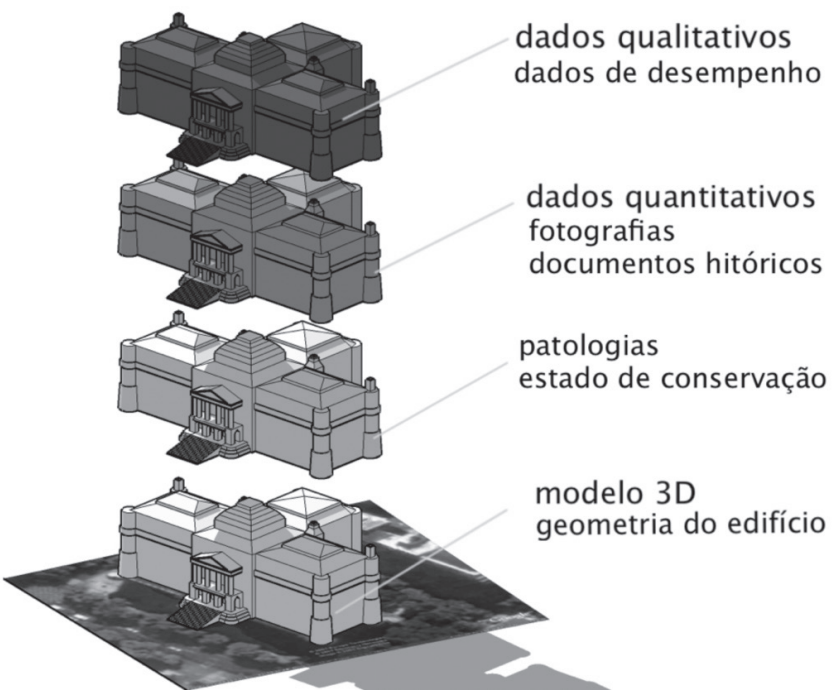

Figura 3: Esquema de Camadas associativas de informações ao modelo 3D da edificação histórica. Elaboração: autora.

\section{Considerações}

Como se trata de uma pesquisa em andamento, ainda não temos elementos suficientes para estabelecer considerações definitivas. O projeto encontra-se na fase de elaboração da categorização dos 
componentes levantados no projeto e na construção da Cia Prada e a criação de uma sistemática para a sua catalogação, que inclui diagramas, esquemas e elementos tridimensionais, elaborados com o recurso de softwares que permitem a modelagem da informação da construção - BIM. Esse material possibilitará uma melhor compreensão das técnicas construtivas e dos componentes empregados nesta edificação, evidenciando estilos, grau de preservação, documentação histórica, etc.

Acreditamos que, em um futuro próximo, estas tecnologias possam ser utilizadas para criar ambientes de análise de dados do edifício que forneçam uma estrutura de integração de coleta, seleção e análise de dados, planejamento e controle das edificações. Esses dados também poderão permitir o desenvolvimento de sistemas de informação, pró-ativo e eficiente, para o gerenciamento de intervenções em edifícios históricos, tanto para para gestores quanto para usuários, visando disseminar informação relativa ao bem patrimonial e sua conservação.

Assim, com esse processo de levantamento, incluindo a modelagem tridimensional, pretende-se criar um material inovador de inventário histórico-documental de edifícios de importância histórica em sistemas de informação baseados no modelo, desempenhando um papel essencial no processo de preservação e manutenção do patrimônio histórico construído.

\section{Agradecimentos}

À CAPES/CNPq e ao SAE/UNICAMP pelas bolsas de Iniciação Científica dos alunos envolvidos neste projeto.

\section{Referências}

BIPS (2008) Digital construction, 3D Working Method, Danish Governmen.

Caldas, C. H., Soibelman, L. \& Gasser, L. (2005). Methodology for the Integration of Project Documents in Model-Based Information Systems. Journal of Computing in Civil Engineering, Vol. 19, No. 1, January, 25-33,

Caldas, C.H., Soibelman, L. (2003). Automating hierarchical document classification for construction management information systems. Automation in Construction, 12(4), 395-406.

Caldas, C.H., Soibelman, L. \& Han, J. (2002). Automated classification of construction project documents. Journal of Computing in Civil Engineering, 16(4), 234-243.

Fai, S., Graham, K., Duckworth, T., Wood, N. \& Attar, R. (2011) Building Information Modelling and Heritage Documentation, 23rd International Symposium, International Scientific Committee for Documentation of Cultural Heritage (CIPA). Prague, Czech Republic.

Kosovac, B., Froese, T. \& Vanier, D. (2000). Integrating heterogeneous data representations in model-based AEC/FM systems. Proceedings of CIT 2000, Reykjavik, Iceland, 1, 556-566.

Manferdini, A.M., Remondino, F. (2012). A Review of Reality-Based 3D Model Generation, Segmentation and Web-Based Visualization Methods. International Journal of Heritage in the Digital Era, v. 1, n. 1 p. 103-124, mar. 2012. Retrieved from: http://multiscience.metapress.com/content//242674440124408/fullt ext.pdf

Noriega, F.M.; Barba, V.G.; Merino, J.A.; Zancajo, J.J. \& Pérez, T.M. (2011). Archilnspection Project: Integrated Non Destructive Testing, A Building Information Model Approach. In: Respecting Fragile Places, 29th eCAADe Conference Proceedings. University of Ljubljana, Faculty of Architecture (Slovenia), 383-392.

Woo, J., Wilsmann, J. \& Kang, D. (2010) Use of As-Built Building Information Modeling. In: Construction Research Congress. Proceedings... Banff: CRC, 2010.

Zhu, Y., Issa, R. R. \& Cox, R. F. (2001). Web-based construction document processing via a malleable frame. Journal of Computing in Civil Engineering, 15(3), 157-169. 